\title{
Design and evaluation of two different finger concepts for body-powered prosthetic hand
}

\author{
Gerwin Smit, MSc, PhD; ${ }^{*}$ Dick H. Plettenburg, MSc, PhD; ${ }^{1}$ Frans C. T. van der Helm, MSc, PhD ${ }^{\mathbf{2}}$ \\ ${ }^{1}$ Delft Institute of Prosthetics and Orthotics, Department of Biomedical Engineering, Delft University of Technology, Delft, \\ the Netherlands; ${ }^{2}$ Department of Biomechanical Engineering, Delft University of Technology, Delft, the Netherlands
}

\begin{abstract}
The goal of this study was to find an efficient method of energy transmission for application in an anthropomorphic underactuated body-powered (BP) prosthetic hand. A pulley-cable finger and a hydraulic cylinder finger were designed and tested to compare the pulley-cable transmission principle with the hydraulic cylinder transmission principle. Both fingers had identical dimensions and a low mass. The only thing that differed between the fingers was the transmission principle. The input energy was measured for a number of tasks. The pulley-cable finger required more input energy than the hydraulic cylinder finger to perform the tasks. This was especially the case in tasks that required high pinch forces. The hydraulic cylinder transmission is therefore the more efficient transmission for application in BP prosthetic fingers.
\end{abstract}

Key words: articulating finger, body-powered, efficiency, hydraulic cylinder finger, hysteresis, prosthetic design, prosthetics, pulley-cable finger, qualitative testing, upper limb.

\section{INTRODUCTION}

For many applications of artificial hands in the field of robotics and prosthetics, it is desirable to have a low hand mass. In the field of prosthetics, a high hand mass is a major cause of prosthetic hand rejection by the user [1]. Artificial hands are often heavy because they have multiple motors placed inside the hand [2-3]. Commercially available articulating prosthetic hands, like the i-Limb Ultra [4] and Bebionic3 [5], use one electric motor for each finger. The number of actuators can be reduced by using the principle of underactuation. An underactuated mechanism has, by definition, more degrees of freedom than actuators [6-7]. The configuration of such a mechanism depends not only on the actuator force but also on the external forces acting on the mechanism, e.g., the force acting on the fingers. Because a person with amputation usually has only one control signal available, just one actuator would be enough to control all finger joints of the entire hand. Using only one actuator could drastically reduce the hand mass. Instead of using an actuator, it is also possible to have the user mechanically control the hand, e.g., by means of a shoulder harness. In such a body-powered (BP) prosthesis, no electric motor is needed, reducing the mass even further.

\section{Problem}

The problem with current BP prosthetic hands is that they require a large amount of input energy by the user to produce a limited pinch force at the fingertip. A BP prosthetic hand requires up to $2,292 \mathrm{Nmm}$ of energy to pinch

\footnotetext{
Abbreviations: $\mathrm{BP}=$ body-powered, DIP = distal interphalangeal, $\mathrm{MCP}=$ metacarpophalangeal, $\mathrm{PCTFE}=$ polychlorotrifluoroethylene, PIP = proximal interphalangeal.

*Address all correspondence to Gerwin Smit, MSc, PhD; Department of Biomechanical Engineering, Delft Institute of Prosthetics and Orthotics, Delft University of Technology, Mekelweg 2, 2628 CD, Delft, the Netherlands; +31152781688. Email: g.smit@tudelft.nl
}

http://dx.doi.org/10.1682/JRRD.2012.12.0223 
$15 \mathrm{~N}$, a user effort that is uncomfortably high [8]. User needs of people with hand amputation include a higher pinch force [9], a lower activation effort [8], and a lower hand mass [10]. To achieve these goals, an efficient energy transmission is required. The input energy can be transmitted to the fingertip through a pulley-cable transmission or hydraulic cylinder transmission. The input energy is required through the compliancy of the components (segments, transmission) and by the friction in the joints, resulting in actuator displacement and force while the contact point of the fingertip itself does not move. The low ratio of the actuator lever over the pinch force lever (the total finger length) results in very high actuator forces. This unfavorable lever ratio directly results from the anthropomorphic dimensions. The problem can be illustrated by the following example. To produce a pinch force of $30 \mathrm{~N}$ at a total finger length of $67 \mathrm{~mm}$, a joint torque of 2,010 Nmm is required in the metacarpophalangeal (MCP) joint (Figure 1). When the activation lever in the MCP joint has a length of $5 \mathrm{~mm}$, a very high activation force of $402 \mathrm{~N}$ is required.

Ideally, the pinched object and the finger parts would be totally rigid. The displacement of the actuator during pinching would then be 0 . Hence, the input energy or work (force $\times$ displacement) would then be 0 , so the actuator does not have to produce input energy ( $\sim$ work). However, in practice, the pinched object and finger parts will be compliant. As a result of the high input force, the object and finger parts will start to elastically deform. To build up a pinch force, the actuator has to produce an input displacement, even when the contact point is not moving. This will result in considerable work by the prosthesis user (Equation (1)):

$$
W=\int_{0}^{l} F(x) \cdot d x
$$

Because of the high activation force $(F(x))$, even a small input displacement $(d x)$ will require a large amount of energy $(W)$. The finger parts act like springs, which store large amounts of input energy. The actuator has to deliver this input energy. Although the stored "elastic energy" is returned during reopening ( $E_{\text {elastic }}$, Figure 2), it is not useful to the BP prosthesis user. This is the first cause of energy loss. A second cause is located in friction in the finger joints. Due to the deflection of the finger parts, there will be small joint rotations. The high activation

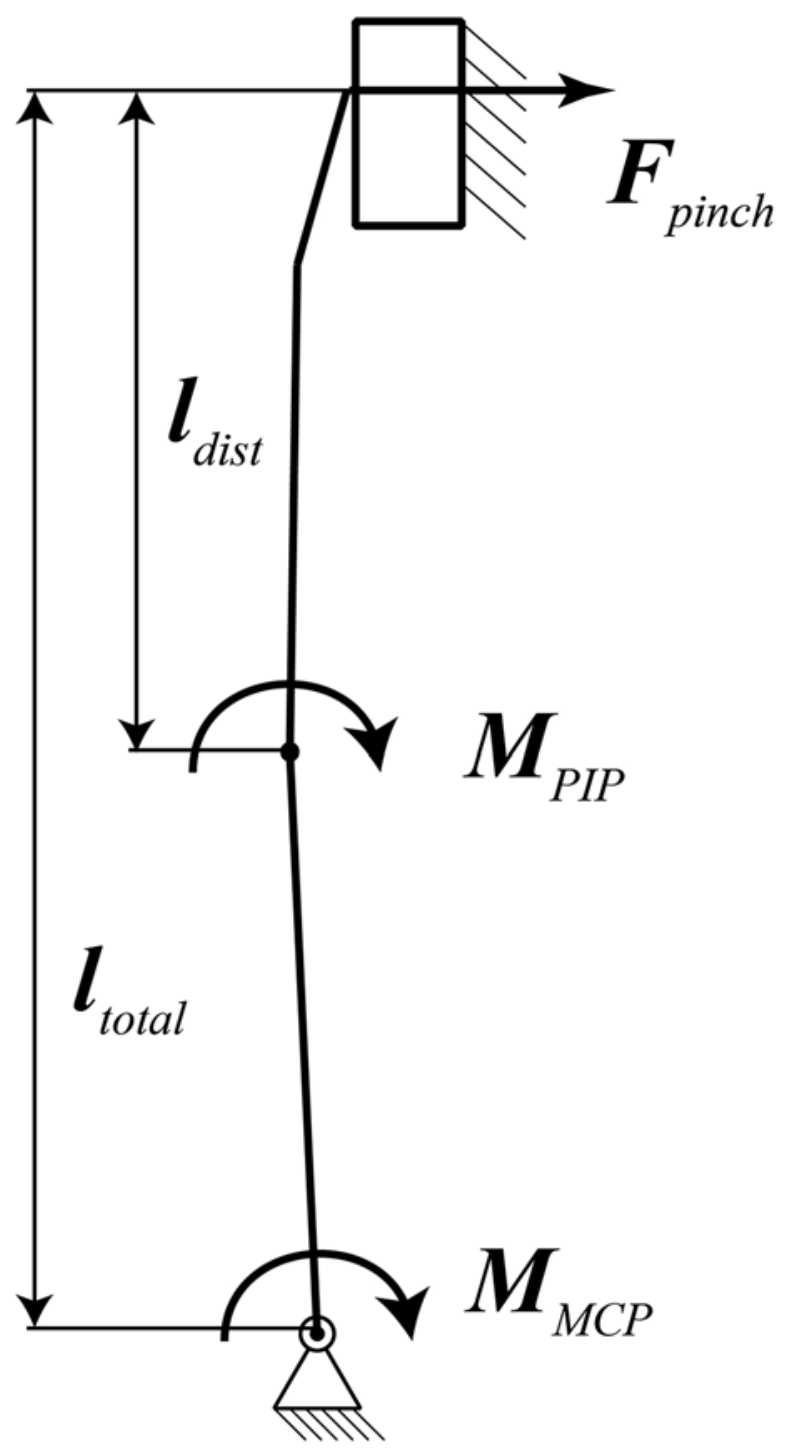

Figure 1.

High activation joint torques are required to produce pinch force $\left(F_{\text {pinch }}\right)$ of $30 \mathrm{~N}$. When total finger length $\left(I_{\text {total }}\right)$ is $67 \mathrm{~mm}$ and distal finger length $\left(I_{\text {dist }}\right)$ is $37 \mathrm{~mm}$, joint torque must be $2,010 \mathrm{Nmm}$ in metacarpophalangeal joint $\left(M_{M C P}\right)$ and $1,110 \mathrm{Nmm}$ in proximal interphalangeal joint $\left(M_{P I P}\right)$.

force causes high joint loads. The rotational friction in the joint will therefore also be high, which results in a considerable energy dissipation even at small joint rotations ( $E_{\text {hys }}$, Figure 2). This energy dissipation caused by friction occurs during the closing and opening motions. The actuator also must deliver this dissipated energy.

Therefore, the design of an efficient mechanism that requires a low amount of input energy ( $E_{\text {close }}$, Figure 2) 
(a)

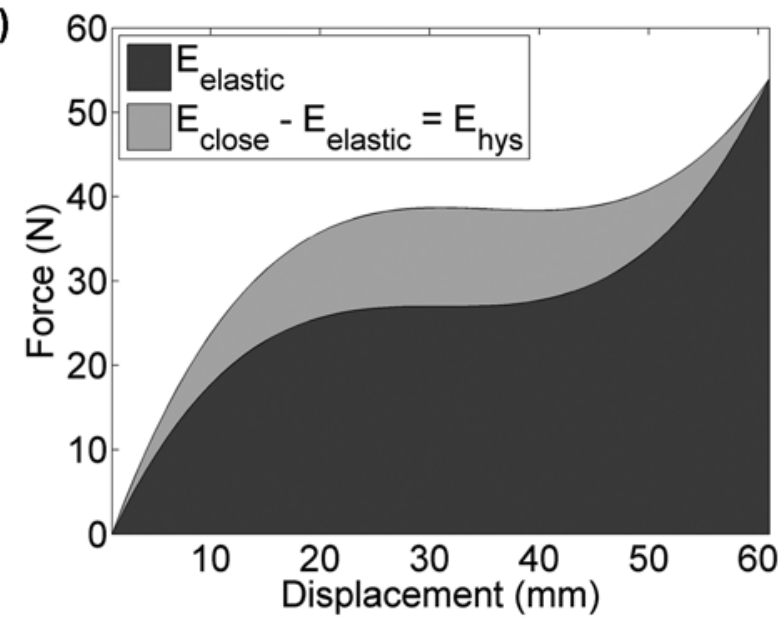

(b)

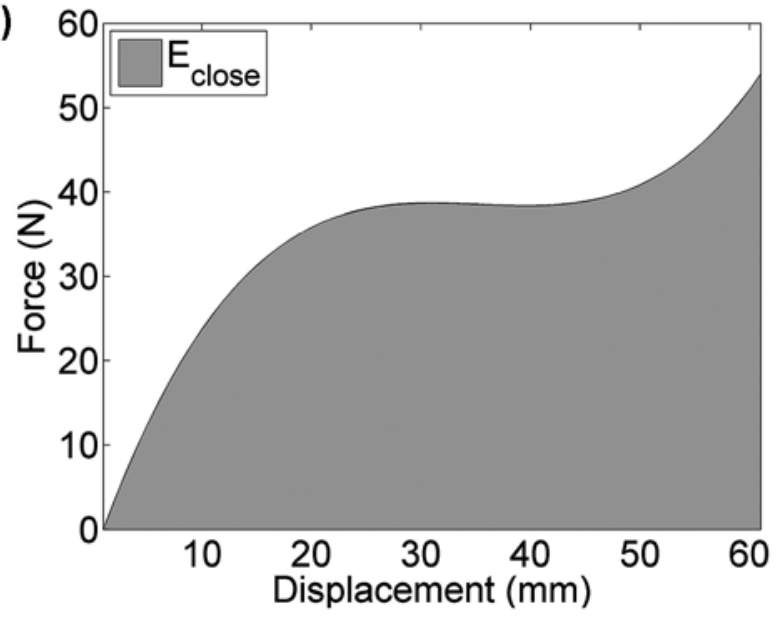

(c)

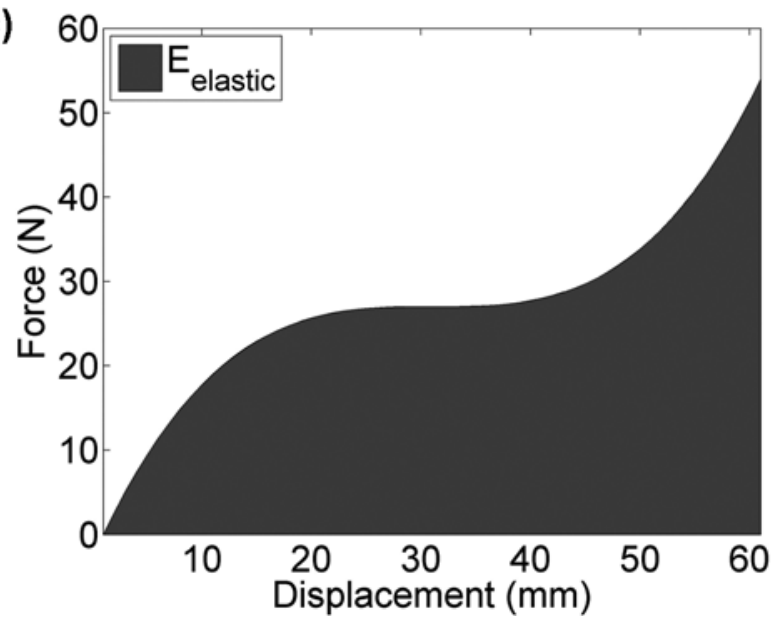

Figure 2.

Work can be represented by area below force-path curve. (a) Hysteresis or energy dissipated by finger $\left(E_{\text {hys }}\right)$ is difference between (b) work done on finger during closing $\left(E_{\text {close }}\right)$ and (c) elastic energy returned by finger during reopening $\left(E_{\text {elastic }}\right)$. Adapted from Smit and Plettenburg [8]. should have a high stiffness (for a low $E_{\text {elastic }}$ ) and should have a low energy dissipation (for a low $E_{\text {hys }}$ ) (Equation (2)):

$$
E_{\text {close }}=E_{\text {elastic }}+E_{\text {hys }} \text {. }
$$

Two recent studies showed that all seven tested BP prosthetic hands, with one degree of freedom, required much input energy, which is already problematic $[8,11]$. Adding more degrees of freedom will increase the number of joints, which will reduce the efficiency even further. When we want to achieve a firm grip with an articulating underactuated prosthetic hand using only a small amount of input energy, we need an efficient energy transmission between actuator and fingertip.

\section{Goal}

The goal of this study was to find an efficient method of energy transmission to enable underactuated articulating finger movement in an anthropomorphic BP prosthetic hand. The mechanism should have a low mass and be able to deliver a requested pinch force with only a small amount of input energy. The pulley-cable finger was compared with the hydraulic cylinder finger in order to select the most efficient transmission principle.

\section{METHODS}

In this study, we compared two different methods of energy transmission: the pulley-cable transmission principle and the hydraulic cylinder transmission principles. Both transmission principles are briefly explained, as well as their advantages and disadvantages.

\section{Pulley-Cable}

A common method of energy transmission is the use of pulleys and cables. This principle has been used in various prosthetic hand prototypes to achieve underactuation [12-13]. Figure 3(a) shows the schematic overview of a pulley-cable transmission in a finger with two degrees of freedom. The pulley at the MCP joint can move independently from the proximal phalanx. The pulley at the proximal interphalangeal (PIP) joint is attached to the distal phalanx. When the cable is pulled, torque is applied to the MCP and PIP joints. The torques are independent of the configuration of the finger. The magnitude of both torques depends on the ratio of the PIP 


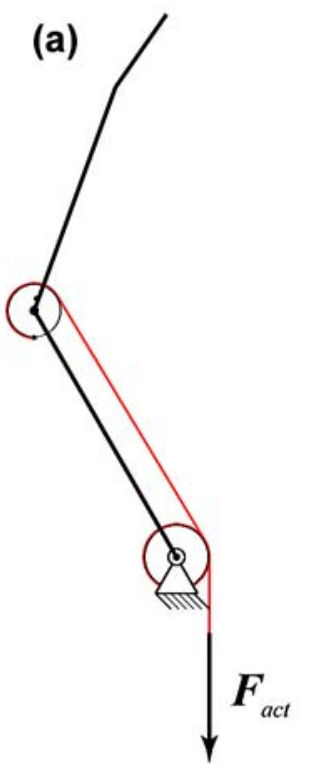

Figure 3.

Schematic drawings of both transmission principles: (a) pulleycable finger and (b) hydraulic cylinder finger. Metacarpophalangeal pulley can move independently, and proximal interphalangeal pulley is attached to distal phalanx. Distal cylinder of hydraulic cylinder finger is located inside finger. Proximal cylinder is located in palm of hand. $F_{\text {act }}=$ activation force, $P_{\text {act }}=$ activation pressure.

pulley diameter to the MCP pulley diameter. The pulleycable transmission has several possible advantages and disadvantages compared with a hydraulic cylinder transmission.

Possible advantages-

- Very lightweight.

- No strict dimension tolerances.

Possible disadvantages-

- Cable wear and tear.

- Cable elasticity.

- Cable runoff.

\section{Hydraulic Cylinder}

Another common method of energy transmission is hydraulics. Although this principle has been used for decades in many fields, only a few examples exist in the field of hand prosthetics [14-18]. Various actuators can be used for hydraulic transmission, e.g., metal bellows, cylinders with O-ring sealing, rolling diaphragms, or
McKibben muscles. In this study, we used hydraulic cylinders with O-ring sealing. Cylinders can withstand higher pressures (>10 MPa) [19] than other hydraulic actuators (varying from $0.8 \mathrm{MPa}$ for McKibben muscles [20] to 6.9 MPa for rolling diaphragms [21]). As a result, cylinders offer the highest force, given a limited crosssectional area. Figure 3(b) shows the schematic overview of the hydraulic cylinder transmission in a finger with two degrees of freedom. A master cylinder, or a hydraulic pump, can pump fluid into the inlet tube. This increases the activation pressure. Due to the increasing activation pressure, the cylinders start to apply a torque around the MCP and PIP joint. The magnitude of both torques depends on the effective cross-sectional area of the cylinders, the lever length, and the lever orientation.

Possible advantages-

- Efficiency independent of hose curvature.

- Flexible to install.

- High system stiffness.

Possible disadvantages-

- Risk of leakage.

- Bulkier than pulley-cable transmission.

- Strict dimension tolerances.

- Sealing friction.

- Large hoses.

- Stiffening up of hoses at high pressures.

\section{Tested Fingers}

We designed two fingers to compare the transmission principle of a pulley-cable finger (Figure 3(a)) with a hydraulic cylinder finger (Figure 3(b)). Except for the transmission principle, all parameters of both fingers were identical (e.g., dimensions, axis diameters, bearings). The fingers had to comply with the following demands:

- Pinch force of $30 \mathrm{~N}$ and handling of a broad range of objects [22].

- Torque ratio between MCP and PIP joint around 0.5 to enable a stable pinch grip [23].

- Anthropomorphic dimensions (fit inside finger of a cosmetic glove size 7 3/4).

- Maximum mass of $25 \mathrm{~g}$ (therefore, maximum mass of four fingers is $100 \mathrm{~g}$ ).

- MCP joint range of $0^{\circ}$ to $90^{\circ}$ (natural range of motion).

- PIP joint range of $0^{\circ}$ to $90^{\circ}$ (natural range of motion). 
- Distal interphalangeal (DIP) joint fixed at $15^{\circ}$ (this angle is used in arthrodesis of the DIP joint) [24].

\section{Designed Fingers}

We designed two fingers, a pulley-cable finger (Figure 4(a)) and a hydraulic cylinder finger (Figure 4(b)). The fingers were identical except for the transmission principle (Table 1). Both fingers had the same dimensions. Each transmission principle was optimized for the test, in such a way that its required input energy, energy dissipation, and mass were all as low as possible. The fingers had to produce high joint torques, e.g., pinching $30 \mathrm{~N}$ with a stretched finger (Figure 1) requires a joint torque of 2,010 Nmm around the MCP joint and a torque of 1,110 Nmm around the PIP joint. This imposed a challenge to the finger designs.

For the pulley-cable finger, a 1 mm-thick cable made of steel was selected. The steel cable has a high stiffness because steel has a high elastic modulus ( 200 GPa). The stiffness of a cable depends on the material stiffness and

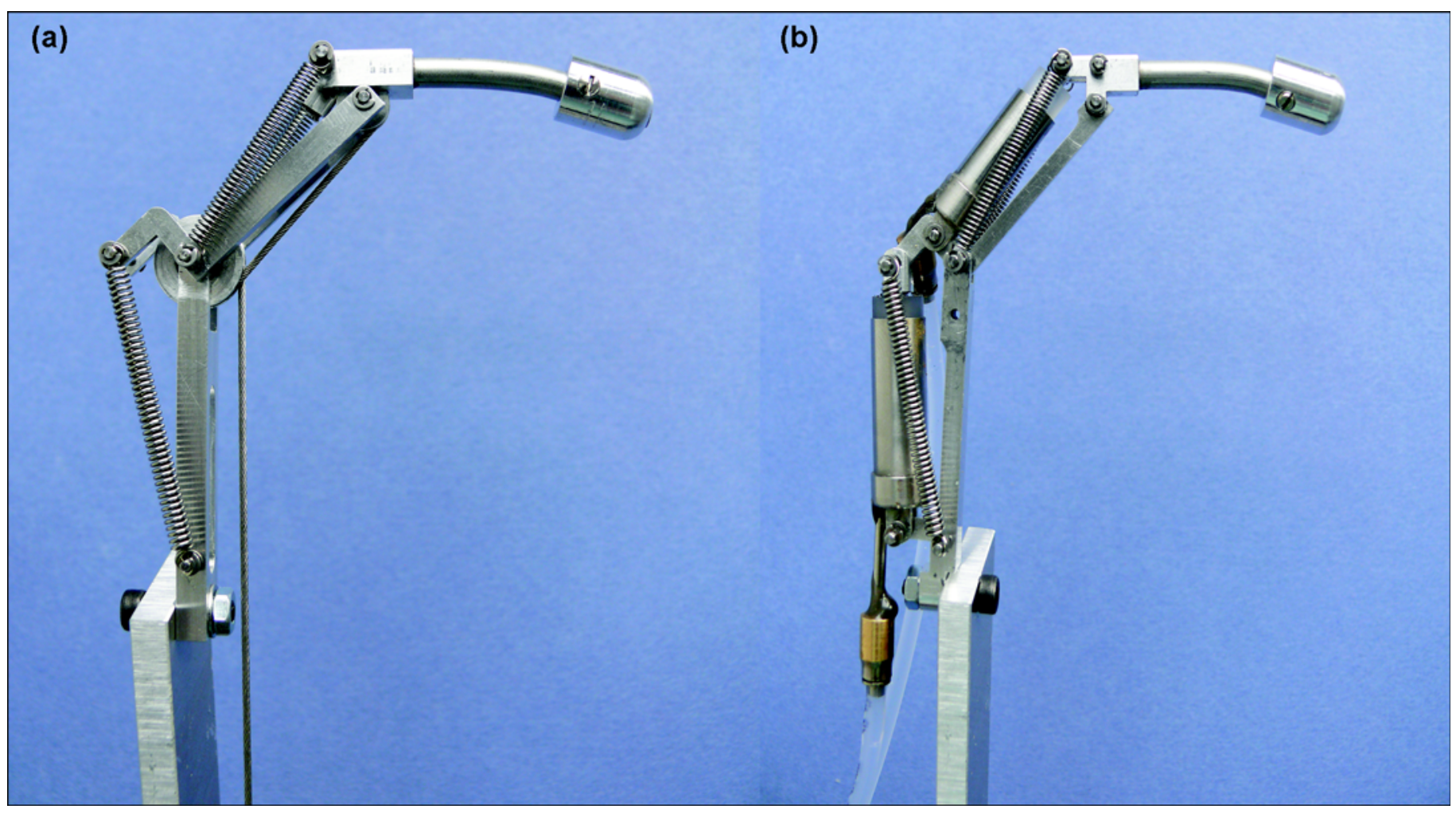

Figure 4.

Two fingers with identical parameters were made: (a) pulley-cable finger and (b) hydraulic cylinder finger. Only parameter that differed was transmission principle. Same springs were used in identical configuration in each finger to extend finger. on the constructional stiffness, which depends on the plait or braid of the cable [25]. Other commonly used high-strength cable materials all have a two to four times lower modulus of elasticity than steel, e.g., Vectran (52103 GPa), Aramid (70-110 GPa), and Spectra/Dyneema (120 GPa). The tension force in the cable of the pulleycable finger had to be minimized to reduce elastic behavior of the cable. A lower cable force will also reduce the bearing load, and thus, the bearing friction. To minimize the cable force, the diameters of the pulleys were maximized. The diameter of the proximal pulley was set at $10 \mathrm{~mm}$, which was the maximal diameter that would fit inside an anthropomorphic finger together with the finger frame. To match the transmission ratio of the hydraulic cylinder finger, the distal pulley diameter had to be 0.55 times the proximal pulley. Given the proximal pulley diameter $\left(d_{2}\right)$ and a cable thickness of $1 \mathrm{~mm}\left(t_{c}\right)$, the distal pulley diameter $\left(d_{1}\right)$ was set at $5 \mathrm{~mm}:\left(\left[d_{1}+t_{c}\right] /\left[d_{2}+t_{c}\right]=\right.$ $[2.5+0.5] /[5.0+0.5]=0.55)$. The finger had a total mass of only $15 \mathrm{~g}$. 
JRRD, Volume 50, Number 9, 2013

Table 1.

Specifications of both finger prototypes.

\begin{tabular}{lcc}
\multicolumn{1}{c}{ Specification } & Pulley-Cable Finger & Hydraulic Cylinder Finger \\
\hline Maximum MPC Joint Angles $\left(^{\circ}\right)$ & 90 & 90 \\
Maximum PIP Joint Angles $\left(^{\circ}\right)$ & 90 & 90 \\
PIP/MCP Ratio & 0.55 & 0.55 \\
Length (mm) & & 67 \\
$\quad$ Total Finger & 67 & 30 \\
Proximal Phalanx & 30 & 37 \\
Middle and Distal Phalanx & 37 & 1.5 \\
Axes Diameter (mm) & 1.5 & 25 \\
Total Finger Mass (g) & 15 & \\
MPC = metacarpophalangeal, PIP = proximal interphalangeal. & \\
\hline
\end{tabular}

The piston diameters of the hydraulic cylinder finger were maximized to enable the high activation forces. The distal cylinder was maximized because there was only limited space to fit the distal cylinder inside the finger. Its diameter was set at $7 \mathrm{~mm}$, which was the largest cylinder diameter that would fit inside the anthropomorphic finger alongside the finger frame. The distal cylinder had a moment arm of $5 \mathrm{~mm}$. There was more space for the proximal cylinder, because it was placed inside the palm of the hand. Its diameter was set at $8 \mathrm{~mm}$. Together with a moment arm of $7 \mathrm{~mm}$, this yields a transmission ratio of 0.55 compared with the distal cylinder. The finger had a mass of $25 \mathrm{~g}$. This is $10 \mathrm{~g}$ more than the mass of the pulley-cable finger. The difference in mass is only 2 percent of the total mass of an average prosthetic hand (which has a mass of $450 \mathrm{~g}$ ). Therefore, both fingers can be considered very lightweight. Flexible nylon hoses $(3.0 \times 1.8 \mathrm{~mm})$ were used, which can withstand a pressure of up to $6 \mathrm{MPa}$. Water was used as a hydraulic fluid instead of oil to limit the consequences in case of a system failure.

Both fingers were unidirectionally activated. After activation, they were returned to their initial position by helical springs. The same springs in the same configuration were used for both fingers. Plain bearings made of polychlorotrifluoroethylene (PCTFE) were used. The diameter of the axis in the fingers was minimized to $1.5 \mathrm{~mm}$ to reduce the joint friction inside the bearings.

\section{Test Protocol}

The fingers were compared using an energy-based approach. The required activation energy and the energy dissipation were measured while the fingers had to perform the following tasks:

1. Pinch $30 \mathrm{~N}$ with a stretched finger (Figure 5(a)).
2. Close the finger $90^{\circ}$, pinch $0 \mathrm{~N}$, and reopen the finger (Figure 5(b)).

3. Close the finger $90^{\circ}$, pinch $30 \mathrm{~N}$, and reopen the finger (Figure 5(b)).

4. Close the finger $180^{\circ}$, pinch $0 \mathrm{~N}$, and reopen the finger (Figure 5(c)).

5. Close the finger $180^{\circ}$, pinch $30 \mathrm{~N}$, and reopen the finger (Figure 5(c)).

To simulate the effect of a cosmetic glove on a prosthetic finger, the tests were repeated with a PVC (polyvinyl chloride) cosmetic glove placed over the finger. All tests were performed four times for each finger type to obtain average values. The energy transmission that requires the lowest amount of energy was selected as the most efficient transmission.

A manually operated test bench (Figure 6) was used to actuate the fingers. The pulley-cable finger was actuated by pulling the actuation cable. The hydraulic cylinder finger was actuated by pulling a master cylinder $\left(d_{\text {piston }}=10 \mathrm{~mm}\right)$. A load cell (Zemic Inc; Santa Fe Springs, California) measured the force acting on the cable or the master cylinder piston. A linear variable differential transfer (P101.200CL100, Positek Limited; Cheltenham, United Kingdom) measured the displacement of the actuation cable or the piston of the master cylinder. The pinch force was measured using a custombuilt pinch strain gauge load cell. The required work and hysteresis were obtained from the measured actuation forces and displacements.

\section{RESULTS}

Figure 7 shows an example of the raw data of a measurement on the pulley-cable finger without a glove, 
(a)

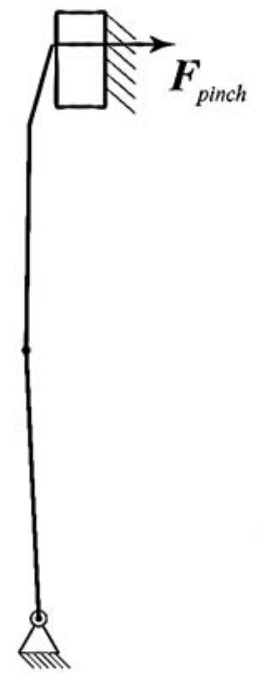

(c)

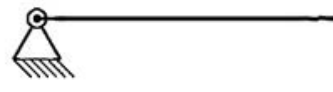

(b)
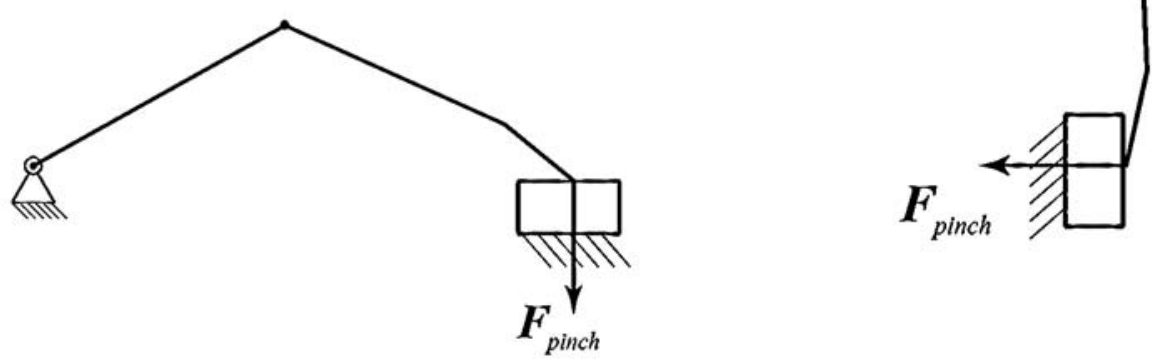

Figure 5.

Schematic overview of tests. (a) Pinch $30 \mathrm{~N}$ in stretched position. (b) Close $90^{\circ}$ and pinch 0 or $30 \mathrm{~N}$. (c) $\mathrm{Close} 180^{\circ}$ and pinch 0 or 30 N. $F_{\text {pinch }}=$ pinch force.

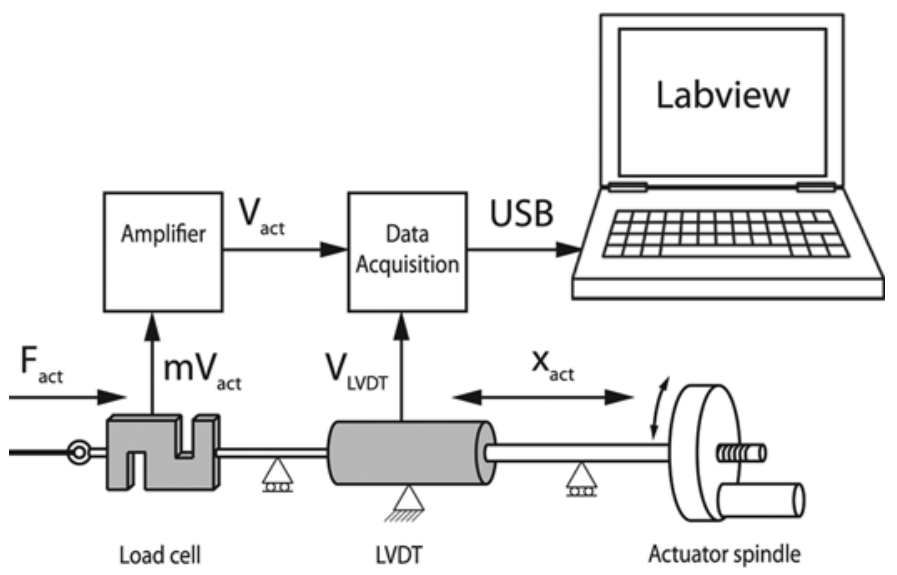

Figure 6.

Schematic overview of manual test bench, which was used to apply load to cable and master cylinder. Load can be applied by turning actuator spindle. Load cell measures actuation force and linear variable differential transfer (LVDT) measures displacement. Adapted from Smit et al. [11]. $F_{\text {act }}=$ force activation cable, $\mathrm{mV}_{\mathrm{act}}=$ voltage force sensor (unamplified), USB = universal serial bus, $V_{\text {act }}=$ voltage sensor (amplified), $\mathrm{V}_{\mathrm{LVDT}}=$ voltage displacement sensor, $\mathrm{x}_{\mathrm{act}}=$ displacement activation cable. bending $90^{\circ}$ and pinching $30 \mathrm{~N}$. The arrows mark the steps: (1) closing $90^{\circ}$, (2) increasing pinch force up to $30 \mathrm{~N}$, (3) unloading the finger, and (4) stretching the finger. The input energy ( $\left.E_{\text {close }}\right)$ is the area below lines 1 and 2 . The returned elastic energy ( $\left.E_{\text {elastic }}\right)$ is the area below lines 3 and 4 . The dissipated energy $\left(E_{\text {hys }}\right)$ is the area enclosed by lines 1, 2, 3, and 4 . Table 2 presents the measured work and hysteresis values of all tests.

\section{Work}

Figure 8 shows the input energy or total work ( $\left.E_{\text {close }}\right)$ for both fingers for every performed test. The required amount of work ranged from 111 to $1,214 \mathrm{Nmm}$ for the pulley-cable finger and from 113 to $1,111 \mathrm{Nmm}$ for the hydraulic cylinder finger. When the cosmetic glove was applied, both fingers required more input energy than without the glove. For tasks that involved only moving, the pulley-cable finger required up to 35 percent more input energy than the hydraulic cylinder finger. For pinching tasks, the pulley-cable finger required up to 74 percent more energy than the hydraulic cylinder finger.

\section{Hysteresis}

Figure 9 shows the dissipated energy or hysteresis $\left(E_{\text {hys }}\right)$ for both fingers for every performed test. The 

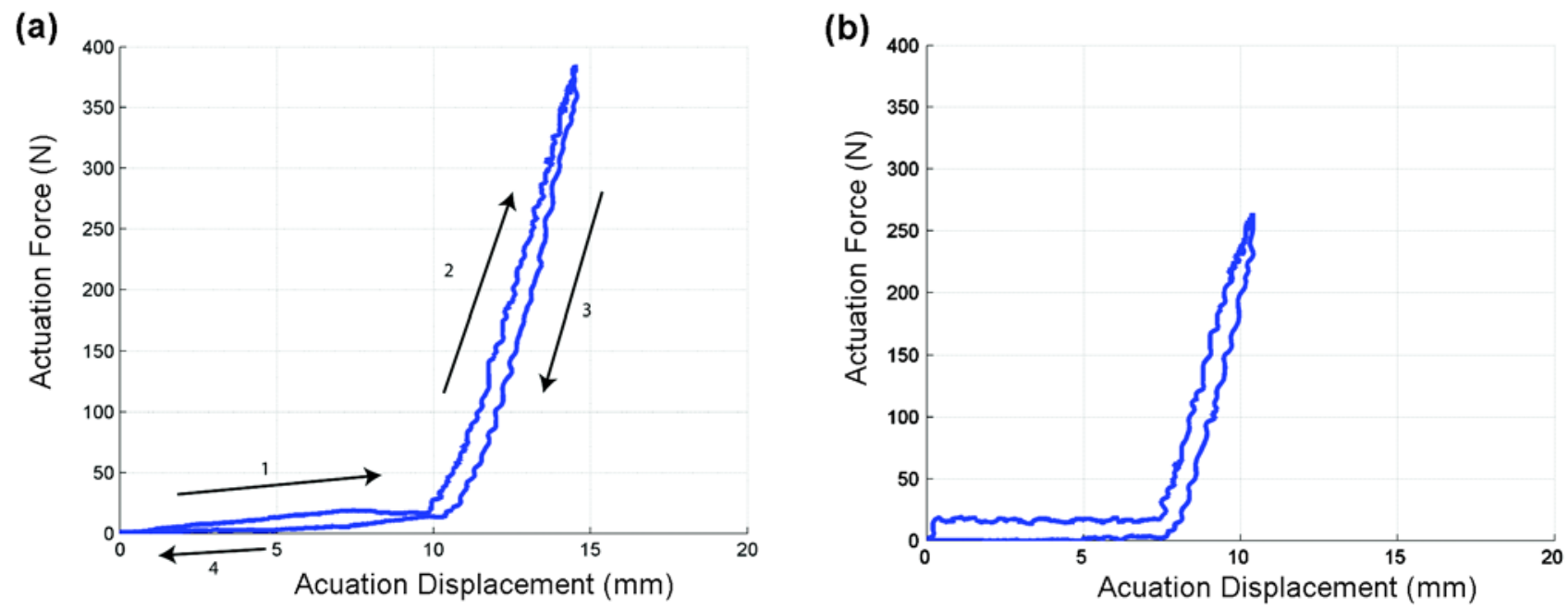

Figure 7.

Example of raw data of (a) pulley-cable finger and (b) hydraulic cylinder finger at $90^{\circ}$ finger flexion, pinching $30 \mathrm{~N}$, without glove. Steps are marked by arrows: (1) closing $90^{\circ},(2)$ increasing pinch force up to $30 \mathrm{~N}$, (3) unloading finger, and (4) stretching finger. In this test, pulley-cable finger requires larger actuation displacement as well as higher actuation force.

Table 2.

Required work and dissipated energy for different tasks.

\begin{tabular}{|c|c|c|c|c|c|}
\hline Finger & Angle $\left({ }^{\circ}\right)$ & Pinch Force (N) & Glove & Work (Nmm) & Hysteresis (Nmm) $^{*}$ \\
\hline Pulley-Cable & 0 & 30 & Frame & $858 \pm 15$ & $214 \pm 15$ \\
\hline Pulley-Cable & 0 & 30 & Frame + Glove & $1,148 \pm 95$ & $431 \pm 70$ \\
\hline Pulley-Cable & 90 & 0 & Frame & $111 \pm 1$ & $58 \pm 1$ \\
\hline Pulley-Cable & 90 & 0 & Frame + Glove & $127 \pm 10$ & $47 \pm 9$ \\
\hline Pulley-Cable & 90 & 30 & Frame & $954 \pm 15$ & $243 \pm 8$ \\
\hline Pulley-Cable & 90 & 30 & Frame + Glove & $1,009 \pm 26$ & $253 \pm 13$ \\
\hline Pulley-Cable & 180 & 0 & Frame & $245 \pm 3$ & $150 \pm 4$ \\
\hline Pulley-Cable & 180 & 0 & Frame + Glove & $486 \pm 45$ & $288 \pm 39$ \\
\hline Pulley-Cable & 180 & 30 & Frame & $1,110 \pm 14$ & $303 \pm 3$ \\
\hline Pulley-Cable & 180 & 30 & Frame + Glove & $1,214 \pm 57$ & $359 \pm 48$ \\
\hline Hydraulic Cylinder & 0 & 30 & Frame & $708 \pm 8$ & $211 \pm 9$ \\
\hline Hydraulic Cylinder & 0 & 30 & Frame + Glove & $761 \pm 7$ & $219 \pm 5$ \\
\hline Hydraulic Cylinder & 90 & 0 & Frame & $113 \pm 2$ & $106 \pm 3$ \\
\hline Hydraulic Cylinder & 90 & 0 & Frame + Glove & $125 \pm 4$ & $115 \pm 4$ \\
\hline Hydraulic Cylinder & 90 & 30 & Frame & $549 \pm 14$ & $251 \pm 7$ \\
\hline Hydraulic Cylinder & 90 & 30 & Frame + Glove & $782 \pm 20$ & $323 \pm 23$ \\
\hline Hydraulic Cylinder & 180 & 0 & Frame & $218 \pm 2$ & $188 \pm 2$ \\
\hline Hydraulic Cylinder & 180 & 0 & Frame + Glove & $360 \pm 19$ & $284 \pm 20$ \\
\hline Hydraulic Cylinder & 180 & 30 & Frame & $990 \pm 19$ & $439 \pm 16$ \\
\hline Hydraulic Cylinder & 180 & 30 & Frame + Glove & $1,111 \pm 35$ & $513 \pm 28$ \\
\hline
\end{tabular}

pulley-cable finger dissipated 47 to $431 \mathrm{Nmm}$. The hydraulic cylinder finger dissipated 106 to $513 \mathrm{Nmm}$. When the cosmetic glove was applied, both fingers dissipated more input energy than without the glove. The hydraulic cylinder finger dissipated up to 51 percent more energy when the glove was applied. During pinching, up to 5.4 times more energy was dissipated in the pulley-cable finger and up to 2.8 times more energy was 


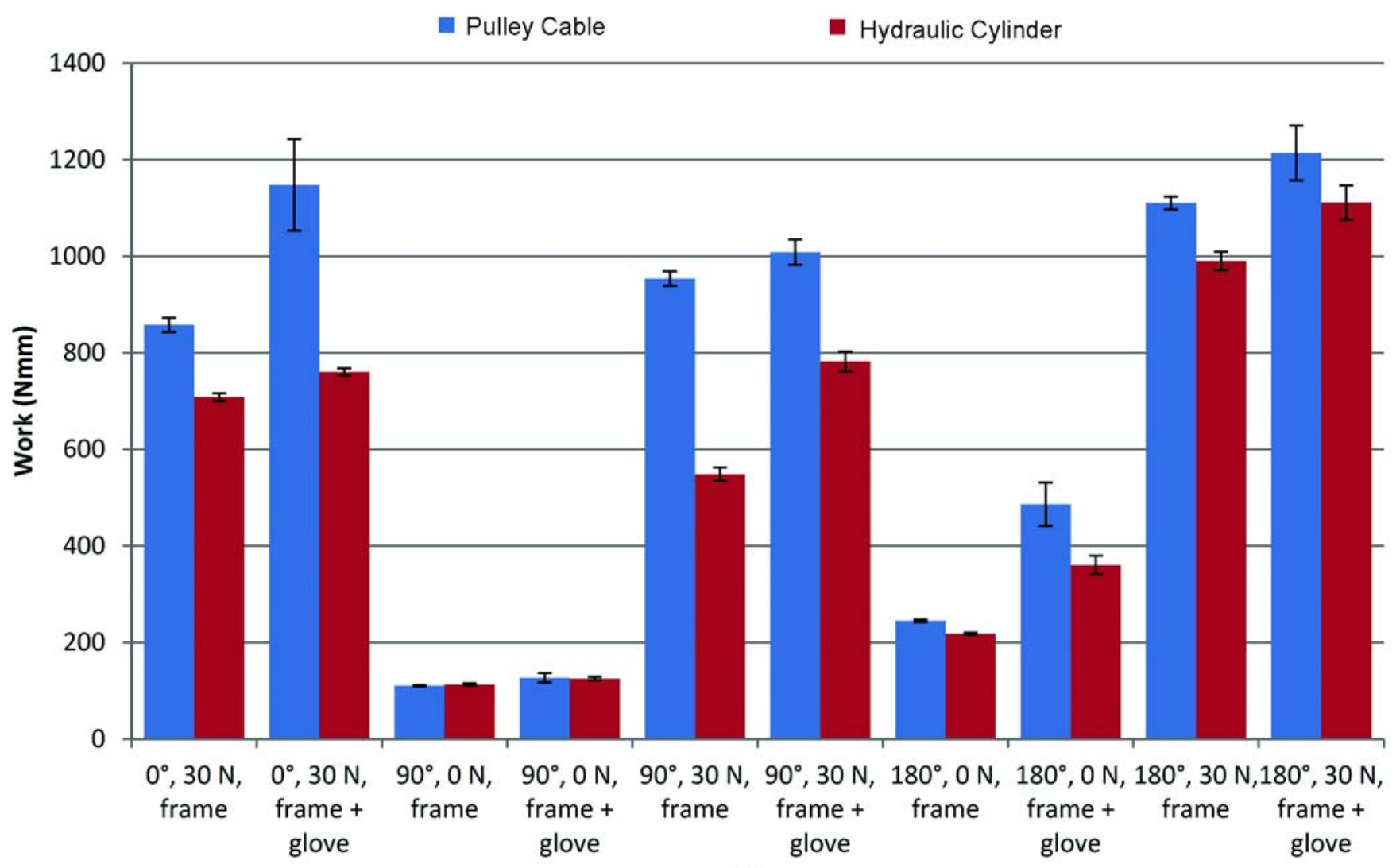

Finger

Figure 8.

Work, or required input energy, to operate fingers during different tasks. Pulley-cable finger required more input energy (or work) than hydraulic cylinder finger to perform same tasks.

dissipated in the hydraulic cylinder finger compared with tasks without pinching.

\section{DISCUSSION}

Based on their dimensions and mass, both fabricated fingers are suitable for application in an anthropomorphic hand prosthesis. The fingers have an anthropomorphic range of motion and are capable of pinching $30 \mathrm{~N}$, which enables a sufficient range of activities and tasks. The mass of each finger ( $\leq 25 \mathrm{~g}$ ) is only 3 to 6 percent of the mass of a current prosthetic hand, which enables the design of a lightweight BP prosthetic hand.

\section{Friction and Hysteresis}

The energy that was dissipated during the tests is dissipated by multiple components. First, there is the bear- ing friction, which increases when the bearing loads increase. To get an idea of the amount of energy dissipation by one bearing, we can estimate the friction in the bearing of the pulley of the MCP joint during $90^{\circ}$ finger flexion and $30 \mathrm{~N}$ pinching without the glove (Figure 7). The joint friction is expected to be the highest when the finger starts pinching. During pinching, the cable force increases linearly (from $\sim 20$ to $\sim 380 \mathrm{~N}$ ), so the average cable force is about $200 \mathrm{~N}$. When we assume that the normal force in the bearing of the MCP pulley is equal to the cable force and the friction coefficient of PCTFE is 0.35 [26], the tangential friction force in the bearing $(d=1.5 \mathrm{~mm})$ will be $70 \mathrm{~N}$. The tangential force at the pulley diameter will be $0.75 \mathrm{~mm} / 5.5 \mathrm{~mm} \times 70 \mathrm{~N}=9.55 \mathrm{~N}$. Because the measured cable displacement during pinching is $5 \mathrm{~mm}$, the bearing will dissipate $5 \mathrm{~mm} \times 9.55 \mathrm{~N}=48 \mathrm{Nmm}$ during closing and the same amount during opening, which yields a total energy dissipation of $95 \mathrm{Nmm}$ per cycle for 


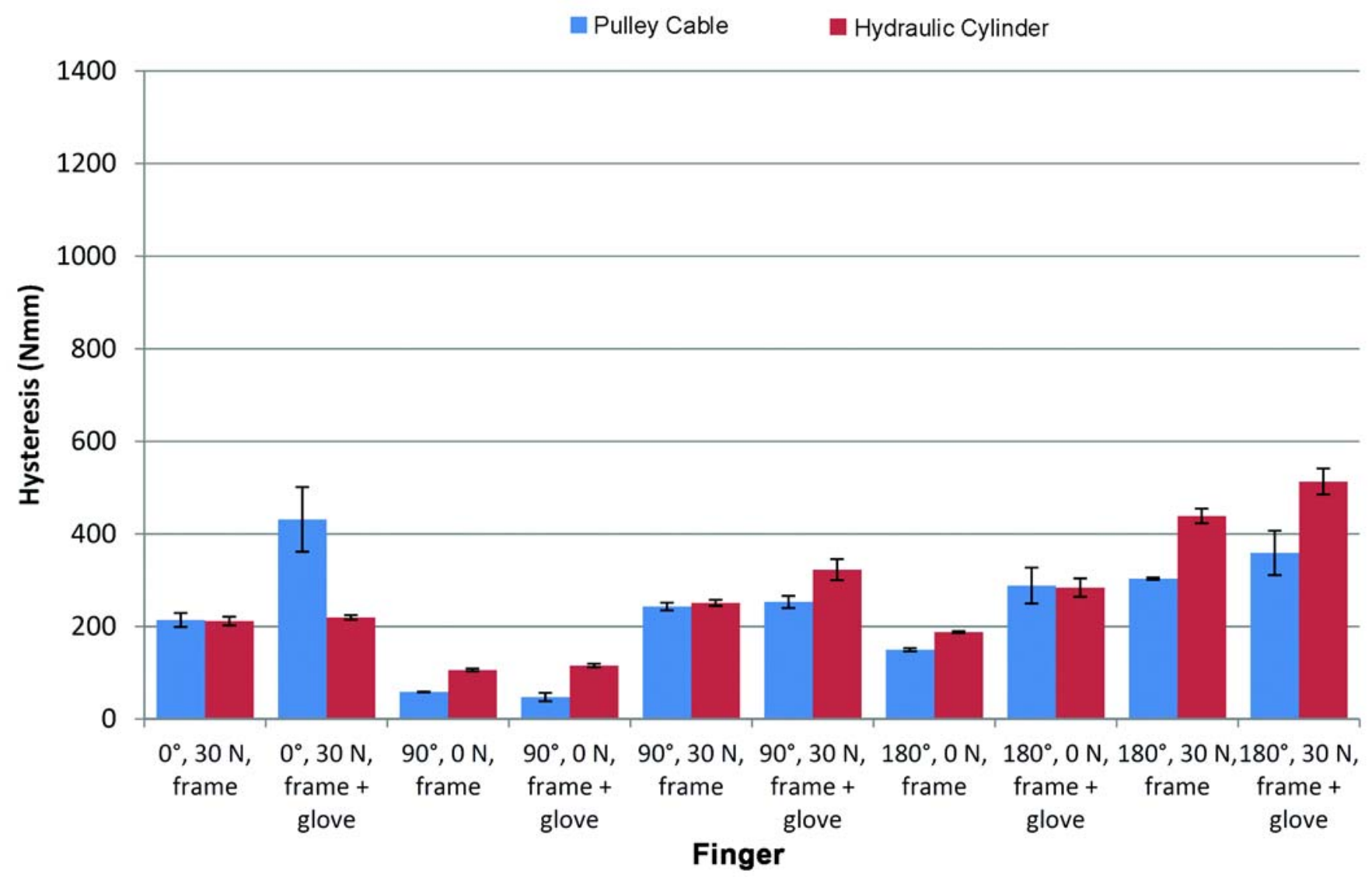

Figure 9.

Hysteresis, or dissipated energy, to operate fingers during different tasks.

the bearing of the MCP pulley. Besides the friction in the MCP pulley, there will also be friction in the MCP and PIP joint. Doubling the pulley diameter would halve the bearing friction. It is, however, not possible to increase the pulley diameter, because then the pulley-cable finger would not fit inside the cosmetic glove.

Second, there is energy dissipation in all components that have viscous behavior, like the cosmetic glove [27]. Therefore, the dissipated energy of both fingers is expected to be lower during movements without pinching or without a counteracting cosmetic glove. This was confirmed by the results (Figure 9). When the glove was applied, the fingers required more input energy. In the hydraulic cylinder finger, an extra cause of extra energy dissipation is the sealing friction, which is caused by the O-ring sliding along the cylinder wall [19]. This friction is always present, even when the piston is moving without external loads acting on the finger. This is a disadvan- tage compared with the pulley-cable finger. The effect could be clearly seen when the fingers were bent $90^{\circ}$ without pinching (Figure 9). In this unloaded test, the hydraulic cylinder finger dissipated significantly more energy than the pulley-cable finger due to the O-ring friction. After the cosmetic glove is applied, the pulley-cable finger shows a remarkable increase of the hysteresis in the stretched configuration. This can possibly be explained by contact friction between the glove and mechanism in this configuration.

\section{System Stiffness and Required Work}

For unloaded movements, the required work mainly depends on the helical springs; the elastic deformation of the glove, if present; and the energy dissipation, as described previously. As soon as the finger must deliver a pinch force, the forces acting on the finger will increase and various components of the finger will start to elastically 
deform and start acting like springs. This is clearly shown in the test in which the stretched fingers pinch $30 \mathrm{~N}$. Although the helical springs are not extended during this test, the fingers still store elastic energy. The amount of deformation of components such as frame, cable, or hose depends on their stiffness. The individual stiffnesses of all the components together determines the total stiffness of the system. The stiffer the system is, the less extra energy is required to deliver the demanded pinch force. Although the components return their elastic energy when returning to their original shape, the returned energy is not useful anymore because it is not possible to return the energy to the user in a useful way. A system with a low stiffness will therefore have a higher energy demand, which will result in extra physical effort for BP prosthetic hand users. Elastic deformation is therefore undesirable. It should be avoided by making the system stiffness as high as possible.

The results show that the pulley-cable finger requires more energy in tasks that involve pinching, even in tasks in which its hysteresis is lower. The pulley-cable finger stores more elastic energy during one cycle, which means that it has a lower stiffness than the hydraulic cylinder finger. A cause of its lower stiffness lies in the elastic behavior of the cable, which elastically elongates when the activation force increases. This effect could be reduced by using a thicker cable or two cables instead of one. However, this is not possible in the designed finger because the limits were already reached during the optimization. Increasing the cable diameter, at a constant pulley radius, will increase the stress in the outer cable filaments, which will result in cable failure. Also, the pulley width is too small for a thicker cable or a double cable. It is also not possible to use a stiffer cable of a material with a much higher Young modulus than the current steel cable, because alternative cable materials, such as Vectran, Aramid, and Spectra/Dyneema, all have a two to four time lower elastic modulus. Therefore, cables made of materials other than steel will be less stiff and require even more energy input. Another cause for the larger elastic deformation of the pulley-cable finger is the high cable force that exerts an external force to the finger frame, causing small elastic deformations of the frame. The hydraulic hose of the hydraulic cylinder finger does not impose an external force on the finger frame of the hydraulic cylinder finger. Less elastic deformation takes place in the frame of the hydraulic cylinder finger because only internal forces act on the frame.

\section{Future Clinical Implications}

The fingers in this study were both able to produce a pinch force of $30 \mathrm{~N}$. This is a high pinch force for an articulating underactuated finger and a 1.7 to 4.3 times higher pinch force than current BP prosthetic hands $[8,11]$. With two fingers, this would enable a tripod grip of $60 \mathrm{~N}$. The hydraulic hose of the hydraulic cylinder finger allows for an increase in pressure and pinch force. The pinch force of the pulley-cable finger cannot be increased because of the limited strength of the actuation cable. Both fingers are lightweight, having a mass of only 3 to 6 percent of the total mass of an average prosthetic hand. When applied in a BP prosthetic hand, no electric actuators are needed, which allows for a low total hand mass. The presented finger concepts enable the construction of an articulating hand that is lighter, can pinch harder, and can have a higher energy efficiency than current BP prosthetic hands, thereby meeting the most important user demands. This enables a breakthrough in the development of BP prosthetic hands in a field that has not changed significantly in the past decades [11]. Of the two tested principles, the finger with the hydraulic cylinder transmission required the least input energy and was therefore selected to be the most efficient transmission principle. An extra benefit of the hydraulic cylinder transmission is that it can also replace the Bowden cable transmission between the hand and user [28].

\section{Study Strengths and Limitations}

To our knowledge, this study is the first to make a quantitative comparison between a pulley-cable transmission and a hydraulic cylinder transmission in anthropomorphic finger design. The focus on energy is important to enable the development of efficient and lightweight BP prosthetic hands. Because the dimensions are the same for both fingers and because the fingers were optimized for the tasks, the study shows a fair comparison between both transmission principles. A limitation of this study is that it focuses on just one finger, while most hands have multiple fingers. Although this is a limitation, it is unlikely that the difference in energy requirement will decrease between both transmission principles when multiple fingers are added. The difference might even increase when extra pulleys and cables are introduced to enable underactuation among the pulley-cable fingers. To enable underactuation among hydraulic cylinder fingers, a simple manifold can be used, which will not introduce major energy losses. The 
designed fingers can also be used in robotic hands, because the constraint of a robotic hand are usually less tight than the constraints in prosthetic application.

\section{CONCLUSIONS}

Two finger prototypes were designed and constructed to quantitatively compare a pulley-cable transmission with a hydraulic cylinder transmission. The fingers were optimized for application in a finger in a cosmetic glove for a prosthetic hand. The fingers have identical dimensions and their mass is only 3 to 6 percent of the total mass of a current prosthetic hand. The pulley-cable finger required up to 35 percent more energy than the hydraulic cylinder finger for tasks that required only joint movement without pinching. Also, the pulley-cable finger required up to 74 percent more energy for moving and pinching of $30 \mathrm{~N}$ for various configurations. The test showed that the hydraulic cylinder finger required the lowest amount of input energy to perform identical tasks because it had the highest system stiffness. Both fingers enable the construction of an articulating BP prosthetic hand that is lighter, can pinch harder, and has a higher energy efficiency than current BP prosthetic hands. Of both concepts, the hydraulic cylinder finger is the most suitable for application in a prosthetic hand because it has a higher energy efficiency than the pulley-cable finger.

\section{ACKNOWLEDGMENTS}

\author{
Author Contributions: \\ Study concept and design: G. Smit, D. H. Plettenburg, \\ F. C. T. van der Helm. \\ Acquisition of data: G. Smit. \\ Analysis and interpretation of data: G. Smit. \\ Drafting of manuscript: G. Smit, D. H. Plettenburg. \\ Critical revision of manuscript for important intellectual content: \\ F. C. T. van der Helm. \\ Study supervision: D. H. Plettenburg, F. C. T. van der Helm.
}

Financial Disclosures: The authors have declared that no competing interests exist.

Funding/Support: This material was unfunded at the time of manuscript preparation.

\section{REFERENCES}

1. Biddiss E, Chau T. Upper-limb prosthetics: Critical factors in device abandonment. Am J Phys Med Rehabil. 2007; 86(12):977-87. [PMID:18090439] http://dx.doi.org/10.1097/PHM.0b013e3181587f6c

2. Belter JT, Dollar AM. Performance characteristics of anthropomorphic prosthetic hands. 2011 IEEE International Conference on Rehabilitation Robotics; 2011 Jun 29-Jul 1; Zurich, Switzerland.

3. Belter JT, Segil JL, Dollar AM, Weir RF. Mechanical design and performance specifications of anthropomorphic prosthetic hands: A review. J Rehabil Res Dev. 2013;50(5): 599-618. [PMID:24013909]

http://dx.doi.org/10.1682/JRRD.2011.10.0188

4. Touch Bionics [Internet]. i-Limb Ultra data sheet. Mansfield (MA): Touch Bionics; 2012. Available from: http:// www.touchbionics.com/media/33326/ma00001a ilimb_ultra_datasheet.pdf

5. Bebionic3 [Internet]. Leeds (United Kingdom): RSL Steeper; 2012. Available from: http://bebionic.com

6. Laliberté T, Gosselin CM. Simulation and design of underactuated mechanical hands. Mechanism Mach Theory. 1998;33(1-2):39-57.

http://dx.doi.org/10.1016/S0094-114X(97)00020-7

7. Birglen L, Gosselin C, Laliberté T. Underactuated robotic hands. Berlin (Germany): Springer; 2008.

8. Smit G, Plettenburg DH. Efficiency of voluntary closing hand and hook prostheses. Prosthet Orthot Int. 2010; 34(4):411-27. [PMID:20849359]

http://dx.doi.org/10.3109/03093646.2010.486390

9. Van der Niet O, Reinders-Messelink HA, Bongers RM, Bouwsema H, Van der Sluis CK. The i-LIMB hand and the DMC plus hand compared: A case report. Prosthet Orthot Int. 2010;34(2):216-20. [PMID:20470060]

http://dx.doi.org/10.3109/03093641003767207

10. Biddiss E, Beaton D, Chau T. Consumer design priorities for upper limb prosthetics. Disabil Rehabil Assist Technol. 2007;2(6):346-57. [PMID:19263565] http://dx.doi.org/10.1080/17483100701714733

11. Smit G, Bongers RM, Van der Sluis CK, Plettenburg DH. Efficiency of voluntary opening hand and hook prosthetic devices: 24 years of development? J Rehabil Res Dev. 2012;49(4):523-34. [PMID:22773256] http://dx.doi.org/10.1682/JRRD.2011.07.0125

12. Laliberté T, Baril M, Guay F, Gosselin C. Towards the design of a prosthetic underactuated hand. Mech Sci. 2010;1(1):19-26. http://dx.doi.org/10.5194/ms-1-19-2010

13. Massa B, Roccella S, Carrozza MC, Dario P. Design and development of an underactuated prosthetic hand. IEEE International Conference on Robotics and Automation; 
2002; Washington, DC. p. 3374-79.

http://dx.doi.org/10.1109/ROBOT.2002.1014232

14. LeBlanc M. Current evaluation of hydraulics to replace the cable force transmission system for body-powered upperlimb prostheses. Assist Technol. 1990;2(3):101-7. [PMID:10149042]

15. Kargov A, Pylatiuk C, Oberle R, Klosek H, Werner T, Roessler W, Schulz S. Development of a multifunctional cosmetic prosthetic hand. IEEE 10th International Conference on Rehabilitation Robotics; 2007 Jun 12-15; Noordwijk, the Netherlands. p. 550-53.

16. Goller H, Lewis DW. Hydraulic body-powered system for prosthetic devices. Bull Prosthet Res. 1969;10(12):156-66.

17. Stevenson DA, Lippay AL. Paper 7: Hydraulic powered arm systems. Proc Inst Mech Eng. 1968;183(10):37-44. http://dx.doi.org/10.1243/PIME_CONF_1968_183_175_02

18. Witte F. An electrohydraulic total arm prosthesis with closed loop hydraulic circuits (Elektrohydraulische Ganzarmprothese mit geschlossenen hydraulischen Kreisläufen). Orthopädie Technik. 1977;11:145-57.

19. Martini LJ. Practical seal design. New York (NY): M. Dekker; 1984.

20. Festo [Internet]. Fluidic muscle. Esslingen am Neckar (Germany): Festo Corporation; 2012. Available from: http://www.festo.com/cms/en_corp/9790.htm

21. Bellofram Corporation [Internet]. Rolling diaphragm design manual. Newell (WV): Bellofram Corporation; 2012. Available from: http://www.marshbellofram.com/ belgas/files/2012/05/design_manual.pdf

22. Boelter LM, Keller AD, Taylor CL, Zahm V. Studies to determine the functional requirements for hand and arm prosthesis. Los Angeles (CA): Department of Engineering, University of California; 1947.

23. Kragten GA, van der Helm FC, Herder JL. A planar geometric design approach for a large grasp range in underactuated hands. Mechanism Mach Theory. 2011;46(8):1121-36. http://dx.doi.org/10.1016/j.mechmachtheory.2011.03.004
24. Lamb DW, Hooper G, Kuczynski K. The practice of hand surgery. Boston (MA): Blackwell Scientific Publications; 1989.

25. Sava Industries Inc [Internet]. Design guide for cable solutions. Riverdale (NJ): Sava Industries Inc; 2005. Available from: http://www.savacable.com/sava_cat.pdf

26. Ensigner Ltd [Internet]. Tecaflon PCTFE. Mid Glam (United Kingdom): Ensinger Ltd; 2012. Available from: http://www.ensinger.ltd.uk/1677.file.dld

27. Herder JL, Cool JC, Plettenburg DH. Methods for reducing energy dissipation in cosmetic gloves. J Rehabil Res Dev. 1998;35(2):201-9. [PMID:9651892]

28. LeBlanc M. Evaluation of cable vs. hydraulic transmission of forces for body-powered arm prostheses. 8th Annual RESNA Conference; 1985; Washington, DC. p. 71-73.

Submitted for publication December 7, 2012. Accepted in revised form May 22, 2013.

This article and any supplementary material should be cited as follows:

Smit G, Plettenburg DH, van der Helm FC. Design and evaluation of two different finger concepts for bodypowered prosthetic hand. J Rehabil Res Dev. 2013; 50(9):1253-66.

http://dx.doi.org/10.1682/JRRD.2012.12.0223

ResearcherID/ORCID: Gerwin Smit, MSc, PhD: B9994-2012; Dick H. Plettenburg, MSc, PhD: C-10782012; Frans C. T. van der Helm, MSc, PhD: C-3321-2011

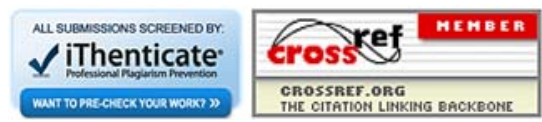


